\title{
Nonlinear Adaptive Parameter Estimation Techniques for Magnetic Transducers Operating in Hysteretic Regimes
}

\author{
James M. Nealis and Ralph C. Smith* \\ Department of Mathematics \\ Center for Research in Scientific Computation \\ North Carolina State University \\ Raleigh, NC 27695
}

\begin{abstract}
Increased control demands in applications including high speed milling and hybrid motor design have led to the utilization of magnetostrictive transducers operating in hysteretic and nonlinear regimes. To achieve the high performance capabilities of these transducers, models and control laws must accommodate the nonlinear dynamics in a manner which is robust with regard to system inputs and facilitates real-time implementation. This necessitates the development of models and control algorithms which utilize known physics to the degree possible, are low-order, and are easily updated to accommodate changing operating conditions such as temperature. We consider here the development of nonlinear adaptive identification techniques for low-order, energy-based hysteresis models having nonlinear parameterizations. We illustrate the techniques in the context of magnetostrictive transducers but they are sufficiently general to be employed for a number of commonly used smart materials including piezoceramics, magnetostrictives and shape memory alloys. The performance of the resulting nonlinear identification algorithms are illustrated through numerical examples.
\end{abstract}

Index Terms: Magnetostrictive materials, hysteresis, constitutive nonlinearities, nonlinear parameterization, adaptive estimation

\section{Introduction}

Piezoceramic, magnetostrictive and shape memory alloy materials are being increasingly considered for use in high performance applications due to their unique transduction capabilities. Piezoceramic transducers are lightweight, exhibit both actuator and sensor capabilities, are highly effective for broadband and high frequency transduction, and have extremely high set point accuracy. These properties are utilized in applications ranging from the design of PZTbased stages for nanopositioning in an atomic force microscope (AFM) [11, 24] to inertial sensors in accelerometers [10]. Magnetostrictive transducers also exhibit broadband transduction capabilities, actuator and sensor capabilities, and the ability to generate large forces while maintaining highly accurate set point placement. This has led to their use as actuators in industrial applications such as high speed milling and highly sensitive sensors for applications such as torque sensing in a steering system [6,21]. Shape memory alloys have the largest work output densities of the three classes of materials but are inherently slower than piezoceramics and magnetostrictive transducers due to the cooling required as a prelude to heat-induced phase transformations. The large strain capabilities of SMA actuators has motivated their use in applications such as active tendon design to attenuate earthquake and wind-induced vibrations in civil structures [25], microgripper design for biomedical instruments [9], and large force generation for reconfigurable airfoil design [13].

While these materials provide unique transduction capabilities, the advantageous performance attributes come at the cost of hysteresis and constitutive nonlinearities throughout most of the drive range. Furthermore, these inherent material properties are dependent upon environmental conditions and system inputs such as temperature, drive frequency, and input or residual stresses - see [16, 23] for quantification of these dependencies in the magnetostrictive transducer compounds Terfenol and Galfenol. To achieve the transducer capabilities in moderate to high

*Email: rsmith@eos.ncsu.edu; Telephone: (919) 515-7552 - Corresponding author 


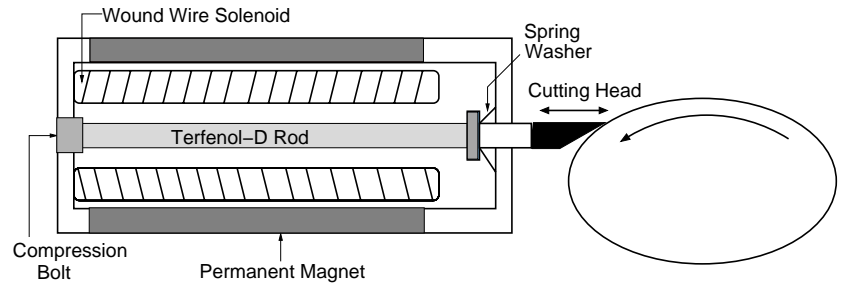

(a)

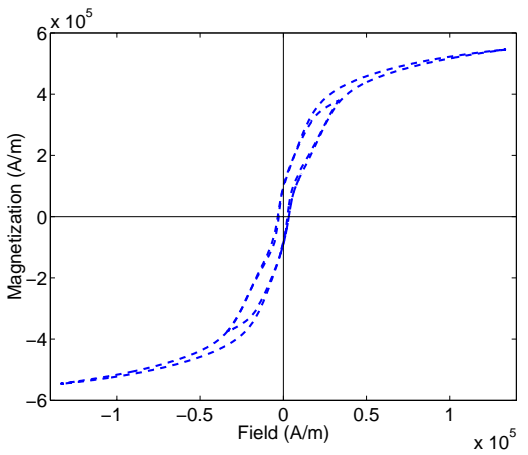

(b)

Figure 1: (a) Prototypical magnetostrictive transducer employed for high speed high accuracy milling. (b) Hysteretic field-magnetization relation as reported in [7].

drive regimes, it is necessary to quantify the hysteresis and constitutive nonlinearities in a manner which is sufficiently accurate to characterize the fundamental nonlinear and hysteretic material behavior and sufficiently efficient to facilitate transducer design and real-time implementation. A fundamental step in the model construction entails the estimation of model parameters given various system inputs. Furthermore, it is often necessary to adaptively update parameters to accommodate the inherent changes in material behavior which occur as environmental conditions change. For example, ohmic heating in a magnetostrictive transducer can significantly affect the hysteretic field-displacement relations exhibited by the transducer whereas Joule heating in an electrostrictive transducer can eliminate the hysteresis if temperature changes are sufficiently large. While these environmental changes often occur on a relatively slow time scale, their effects on the model must be accommodated to maintain the fecundity of the transducer. In this paper, we develop nonlinear parameter estimation techniques in the context of energy-based models which have been employed to quantify the hysteresis and constitutive nonlinearities inherent to magnetostrictive, piezoceramic, and shape memory alloy transducers.

To focus the discussion, we consider a prototypical magnetostrictive transducer employed for applications including high speed, high accuracy milling as depicted in Figure 1a. Input forces and displacements are provides by the Terfenol-D rod in response to fields generated by the surrounding solenoid due to an applied current. As detailed in $[4,7,8]$, these transducer designs have the capacity for generating broadband, high force responses. However, they also exhibit varying degrees of hysteresis and constitutive nonlinearities in the relations between input fields $H$ and the generated magnetization $M$ and strains $e$ as illustrated in Figure 1b as detailed in [7]. Moreover, these nonlinear and hysteretic relations are temperature-dependent and thus change as the transducer heats during the milling process. This necessitates the development of adaptive parameter estimation techniques to update models to maintain the specified cutting accuracy during the milling process.

While this application is quite specific, it illustrates a number of the issues shared by magnetostrictive, piezoceramic and shape memory alloy transducers operating at moderate to high drive levels in variable temperature, variable stress operating conditions. Furthermore, it will be demonstrated that the modeling framework and nonlinear parameter estimation techniques are sufficiently general to permit direct extension to a wide range of piezoceramic and SMA devices.

There exist a number of techniques for modeling hysteresis in ferromagnetic and magnetostrictive materials including micromagnetic relations [3, 12], Preisach representations [1, 22, 32], domain wall models [4, 7, 8, 14, 15], and homogenized free energy relations [27]. Whereas micromagnetic theory addresses fundamental mechanisms associated with magnetostriction, its complexity presently precludes its use in real-time control systems. Preisach models have the advantage of a general and rigorous mathematical framework and can be linearly parameterized in terms of coefficients to be identified, thus permitting the use of linear adaptive estimation and control algorithms [32]. These advantages are offset, however, by the large number of nonphysical parameters required for general operating regimes and the theoretical extensions required to accommodate frequency, stress and temperature dependence. The domain wall models are low-order and have a small number of parameters (e.g., 5-8) which can be correlated with physical properties of the data. Moreover, the methodology is sufficiently general to encompass ferroelectric and piezoceramic materials $[28,30]$ and ferroelastic and shape memory compounds [19] so it provides a unified framework for quantifying hysteresis and constitutive nonlinearities in a broad range of ferroic compounds [29]. For these reasons, we employ a domain wall model to quantify the field-strain behavior depicted in Figure 1b. However, the models 
exhibit a nonlinear dependence on parameters which motivates the development of nonlinear adaptive estimation techniques to accommodate the temperature-dependent hysteresis behavior exhibited by the materials.

In this paper, we extend the techniques of $[17,18]$ to obtain nonlinear adaptive estimation laws for updating parameters in the domain wall models. Among the advantages of the parameter estimation algorithms presented here are the properties that they can be implemented during the operation of the actuator or sensor and require no additional data collection as required for offline least squares algorithms. One possibility is to employ the parameter estimation algorithm during actuator operation to identify parameters during the initialization of the milling process as a prelude to control. Since the behavior of the materials is affected by changing operating conditions, such as temperature, the estimation algorithm can be used to re-identify parameters in the transducer model to reflect changes in the material behavior.

A significant difficulty in developing nonlinear parameter adaptation laws is the fact that gradient update methods are not always sufficient for estimating nonlinearly occurring parameters. To illustrate, consider an error model of the form

$$
\dot{e}=-\lambda e+f(\phi, \theta)-f(\phi, \widehat{\theta})
$$

where $f$ is a nonlinear, scalar-valued function, $\phi$ denotes measured states, $\theta$ and $\widehat{\theta}$ respectively denote a nonlinearly occurring vector of parameters and their estimate, $\lambda>0$ is a scalar, and $e$ is the error between desired and measure trajectories. Consider the gradient update law

$$
\dot{\hat{\theta}}=e \nabla f_{\widehat{\theta}} .
$$

With the standard Lyapunov function, $V=\frac{1}{2}\left(e^{2}+\tilde{\theta}^{2}\right)$, where $\tilde{\theta}=\widehat{\theta}-\theta$, we see that

$$
\dot{V}=-\lambda e^{2}+e\left[f(\phi, \theta)-f(\phi, \widehat{\theta})+\tilde{\theta} \nabla f_{\widehat{\theta}}\right] .
$$

If $e<0$, it is necessary that $\nabla f_{\widehat{\theta}}(\theta-\widehat{\theta}) \leq f(\phi, \theta)-f(\phi, \widehat{\theta})$ which is satisfied if $f$ is convex. If $e>0$, then $\dot{V} \leq 0$ is ensured if $f$ is concave. Hence, the gradient method does not ensure stability for all $\widehat{\theta}$. Moreover, a gradient method applied to a nonlinear parameterized system may not only be insufficient but may lead to instability. The methods we discuss here do not strictly rely on a gradient rule but differ depending on the sign of the error as motivated by (1).

In Section 2, we summarize the hysteresis and transducer models used to characterize the magnetostrictive transducer depicted in Figure 1a. In Section 3 we review the nonlinear adaptive method for the scalar case given in $[17,18]$ and present the extension of this theory to a vector system as is required for the Terfenol-D transducer. Section 4 provides numerical examples of a nonlinear parameter estimation for both the scalar and vector cases.

\section{Actuator Model}

In this section, we construct models for the magnetostrictive transducer which can be employed in the nonlinear parameter estimation algorithm given in Section 3. We develop these models in two steps; (i) the construction of constitutive models which quantify the inherent hysteresis and nonlinearities in the magnetostrictive material, and (ii) the incorporation of the constitutive relations into transducer models which specify the device displacement outputs due to specified current inputs.

\subsection{Nonlinear Hysteretic Constitutive Models}

we describe here a domain wall model quantifying the hysteretic relationship between an applied magnetic field $H$ and the magnetization $M$ in a Terfenol-D rod. The premise of this model is that one source of hysteresis in ferromagnetic materials can be attributed to the impediment of motion of domain walls that are pinned at defects or inclusions in the material. We provide here those details of the theory pertinent to the subsequent development of nonlinear adaptive estimation techniques and refer the reader to $[14,15]$ for details regarding the development of the theory for general ferromagnetic materials and $[4,7,8]$ for the extension of the theory to magnetostrictive transducers.

The domain wall model for the magnetization $M$ produced by an applied magnetic field $H$ will be developed in three steps; (i) quantification of the anhysteretic magnetization $M_{a n}$, (ii) quantification of the irreversible magnetization $M_{i r r}$, and (iii) quantification of the total magnetization $M$.

Physically, $M_{a n}$ can be interpreted as the equilibrium magnetization obtained in the absence of material inclusions. Mathematically, the anhysteretic magnetization can be modeled by quantifying the probability of achieving specified 
energy states through Boltzmann relations. As detailed in $[27,31]$ - or [28] for the analogous ferroelectric model the assumption that magnetic moments can orient only in the direction of the applied field, or diametrically opposite to it, yields the Ising relation

$$
M_{a n}=M_{s} \tanh \left(\frac{H_{e}}{a}\right) .
$$

Alternatively, if one assumes that moments have equal probability of orienting in any direction, one obtains the Langevin model

$$
M_{a n}=M_{s}\left(\operatorname{coth}\left(\frac{H_{e}}{a}\right)-\frac{a}{H_{e}}\right)
$$

which agrees with (2) through first-order terms - see also [5]. In both models, $M_{s}$ denotes the saturation magnetization and

$$
H_{e}=H+\alpha M
$$

quantifies the effective fields where $\alpha$ quantifies denotes a coupling coefficient. A Boltzmann derivation yields the relation

$$
a(T)=\frac{H_{h} T}{T_{c}}
$$

where $H_{h}$ is a biasing field and $T_{c}$ denotes the Curie temperature. Whereas (4) quantifies the temperature-dependence of $a$ in certain operating regimes, the predictions that it provides should typically be considered as qualitative rather than quantitative in nature. Hence, the relation (4) is best interpreted as indicating that temperature-dependence should be accommodated in some manner - e.g., adaptive estimation techniques - rather than providing an accurate model quantifying the temperature behavior.

The anhysteretic relations can be utilized to approximate the magnetization at low drive levels but they do not incorporate the energy loss due to the movement of domain walls and thus can not model high drive levels. To quantify the total magnetization, it is necessary to incorporate the reversible magnetization $M_{r e v}$ and irreversible magnetization $M_{i r r}$. Reversible magnetization occurs when applied field levels are sufficiently small that domain walls remain pinned while the irreversible component represents the movement of pinning sites which occurs at higher drive levels. As detailed in $[8,15], M_{i r r}$ can be quantified as the anhysteretic component minus the losses required to break pinning sites

$$
M_{i r r}=M_{a n}-k \frac{\partial M_{i r r}}{\partial H_{e}}
$$

where $k$ is a measure of the average energy required to translate the domain wall. This relation can be reformulated in terms of the applied field as

$$
\frac{\partial M_{i r r}}{\partial H}=\widehat{\delta} \frac{M_{a n}-M_{i r r}}{k \delta-\alpha\left(M_{a n}-M_{i r r}\right)}
$$

where $\delta=\operatorname{sign}(d H)$ ensures that pinning opposes change in magnetization. The switch $\widehat{\delta}$ is 0 if $d H>0$ and $M>M_{a n}$ or $d H<0$ and $M<M_{a n}$ and 1 otherwise. This is necessary to model the physical observation that after a field reversal, the changes in magnetization are purely reversible until the anhysteretic value is reached.

The reversible magnetization is given by the algebraic relationship

$$
M_{\text {rev }}=c\left(M_{a n}-M_{i r r}\right)
$$

where $c$ is a material parameter which quantifies the reversibility of the material. The total magnetism is the sum of the reversible and irreversible magnetizations which yields

$$
M=(1-c) M_{i r r}+c M_{a n} .
$$

The relation (5) can be reformulated as a differential equation

$$
\begin{gathered}
\frac{\partial M}{\partial H}=F(H, M) \\
M\left(H_{0}\right)=M_{0}
\end{gathered}
$$

where

$$
F(H, M)=\frac{1}{1+c \alpha \frac{\partial M_{a n}}{\partial H}}\left[\widehat{\delta} \frac{M_{a n}-M}{k \delta-\widehat{\alpha}\left(M_{a n}-M\right)}+c \frac{\partial M_{a n}}{\partial H}\right]
$$


with $\widehat{\alpha}=\frac{\alpha}{1-c}$. The anhysteretic magnetization $M_{a n}$ can be taken as either the Ising model (2) or the Langevin model (3).

The anhysteretic behavior modeled by the Ising relation (2) and total magnetization specified by (5) or (6) are illustrated in Figure 2. The parameter values are $a=7012 \mathrm{~A} / \mathrm{m}, k=400 \mathrm{~A} / \mathrm{m}, \alpha=-0.1, M_{s}=7.65 \times$ $10^{5} \mathrm{~A} / \mathrm{m}$ and $c=0.18$. which were determined in [8] through a least squares fit to data measured under isothermal conditions at room temperature. We point out that the model exhibits a nonlinear dependence on the parameters $k$ and $c$ arising in $M_{i r r}$ and $M_{r e v}$ as well as the parameters $M_{s}, \alpha$ and $a$ arising in $M_{a n}$. This necessitates the development of nonlinear adaptive identification techniques to update parameter values online to accommodate changing environmental conditions such as temperature.

\subsection{Transducer Models}

The models (5) or (6) quantify the nonlinear hysteretic relationship between the imposed field and the resulting magnetization in a magnetostrictive material. However, the magnetoelastic coupling which provides the materials with magnetostrictive properties has not yet been addressed. In applications where stress anisotropies dominate crystalline anisotropies, experimental data [8] suggests a quadratic dependence of strain on magnetization. This can be theoretically justified by noting that in present transducer designs, the prestress levels are sufficient to allow stress anisotropies to dominate crystalline anisotropies so that the strains are primarily due to the quadratic rotation processes. To incorporate this quadratic dependence the stress-induced in the Terfenol-D rod is specified as

$$
\sigma=Y e-Y \gamma M^{2}
$$

where $Y$ denotes Young's modulus and $\gamma$ is a magnetoelastic coupling coefficient. This relationship quantifies the linear Hooke's relationship between stresses $\sigma$ and strains $e$ in the Terfenol-D rod as well as the nonlinear hysteretic dependence of the stresses on applied fields $H$ through the magnetization $M$.

The relation (7) quantifies the local elastic behavior of the Terfenol-D rod but does not include spatial dependence nor internal damping in the transducer. We now develop models which specify the displacements and forces generated by the magnetostrictive transducer due to an input current $I(t)$. A partial differential equation model developed in [8] and the corresponding ordinary differential equation (ODE) system derived through a finite element discretization are first summarized. We then demonstrate that the ODE system can be adequately approximated by a second-order scalar differential equation for transducers which employ flux shaping techniques to minimize end effects.

The left end of the Terfenol-D rod $(x=0)$ is assumed to be fixed while the right end $(x=L)$ is constrained by a damped oscillator and has an attached point mass, as depicted in Figure 3. The Kelvin-Voigt damping coefficient and density of the Terfenol-D rod are denoted by $c_{D}$ and $\rho$, and the point mass is denoted by $M_{L}$. The damping spring has stiffness $k_{L}$ and damping coefficient $c_{L}$. Strains in the rod are specified in terms of the longitudinal rod displacement $w$ by $e=\frac{\partial w}{\partial x}$.

The direct use of the constitutive relation (7) will yield an undamped model for the Terfenol-D rod. We assume that stress is proportional to a linear combination of strain and strain rate to incorporate Kelvin-Voigt damping .

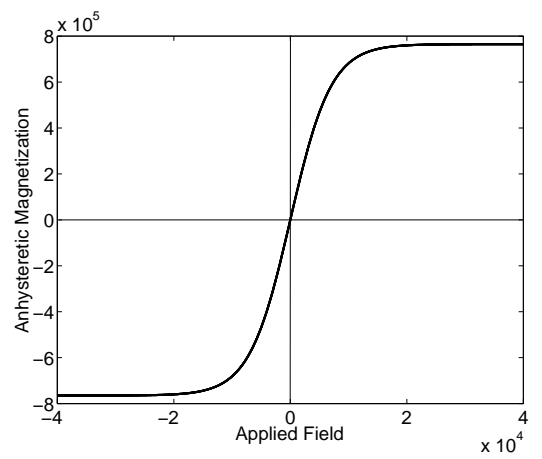

(a)

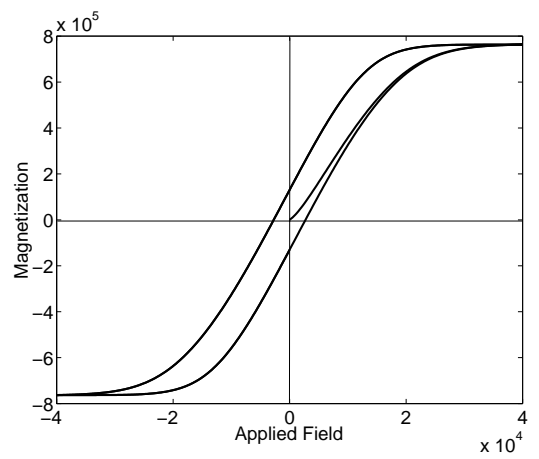

(b)

Figure 2: (a) Anhysteretic magnetization given by (2), and (b) total magnetization specified by (5) or (6). 


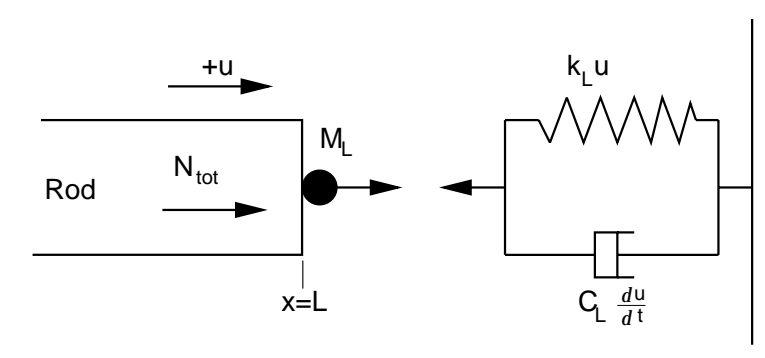

Figure 3: Rod model for Terfenol-D transducer.

Thus, the stress at any point $x, 0 \leq x \leq L$, is given by

$$
\sigma(t, x)=Y \frac{\partial w}{\partial x}(t, x)+c_{D} \frac{\partial^{2} w}{\partial x \partial t}(t, x)-Y \gamma M^{2}(t, x) .
$$

The relation (8) is identical to the relation obtained in [8] if the coupling coefficient is defined as $\gamma=\lambda_{s} / M_{s}$ where $\lambda_{s}$ and $M_{s}$ respectively denote the saturation magnetostriction and saturation magnetization, respectively.

Balancing forces yields

$$
\rho A \frac{\partial^{2} w}{\partial t^{2}}=\frac{\partial N_{t o t}}{\partial x}
$$

where $A$ is the cross sectional area of the Terfenol-D rod and the force resultant is specified by

$$
N_{t o t}(t, x)=Y A \frac{\partial w}{\partial w}(t, x)+c_{D} A \frac{\partial^{2} w}{\partial x \partial t}(t, x)-Y A \gamma M(t, x)^{2} .
$$

To obtain appropriate boundary conditions, we first note that $w(t, 0)=0$. Balancing forces at $x=L$ yields

$$
N_{t o t}(t, L)=-k_{L} w(t, L)-c_{L} \frac{\partial w}{\partial t}(t, L)-M_{L} \frac{\partial^{2} w}{\partial x \partial t}(t, L) .
$$

Initial conditions are taken to be $w(0, x)=0$ and $\frac{\partial w}{\partial x}(0, x)=0$.

As detailed in [8], formulation of the model (9) in weak form and spatial discretization utilizing linear finite elements yields the vector ODE system

$$
\begin{gathered}
\mathcal{M} \ddot{\vec{x}}+\mathcal{C} \dot{\vec{x}}+\mathcal{K} \vec{x}=\gamma \mathcal{B}\left[M^{2}(H)\right](t) \\
\vec{x}(0)=\vec{x}_{0}, \quad \dot{\vec{x}}(0)=\vec{x}_{1}
\end{gathered}
$$

where $\vec{x}(t) \in \mathcal{R}^{N}$ specifies the states, $\mathcal{M} \in \mathcal{R}^{N \times N}, \mathcal{C} \in \mathcal{R}^{N \times N}, \mathcal{K} \in \mathcal{R}^{N \times N}$ respectively denote the mass, damping and stiffness matrices, and $\mathcal{B} \in \mathcal{R}^{N}$ contains integrated basis functions resulting from the inputs. For general systems, large $N$ (e.g., $N=32$ ) may be required to achieve convergence. This may limit the speed at which model-based control designs can be implemented.

For transducer design in which the permanent magnet is constructed to minimize end effects, measurements with a Hall probe indicate that nearly uniform fields

$$
H(t, x)=n I(t)
$$

are achieved along the length of the rod - here $n$ denotes the number of coils per unit length in the solenoid. In such cases, each finite element section of the rod reacts identically to the uniform magnetic field which motivates the consideration of a lumped scalar model

$$
\begin{gathered}
m \ddot{x}+c \dot{x}+k x=\zeta\left[M^{2}(H)\right](t) \\
x(0)=x_{0} \quad \dot{x}(0)=\dot{x}_{0}
\end{gathered}
$$

where $m, c, k$ and $\zeta$ are parameters which must be estimated for a given transducer construction. 

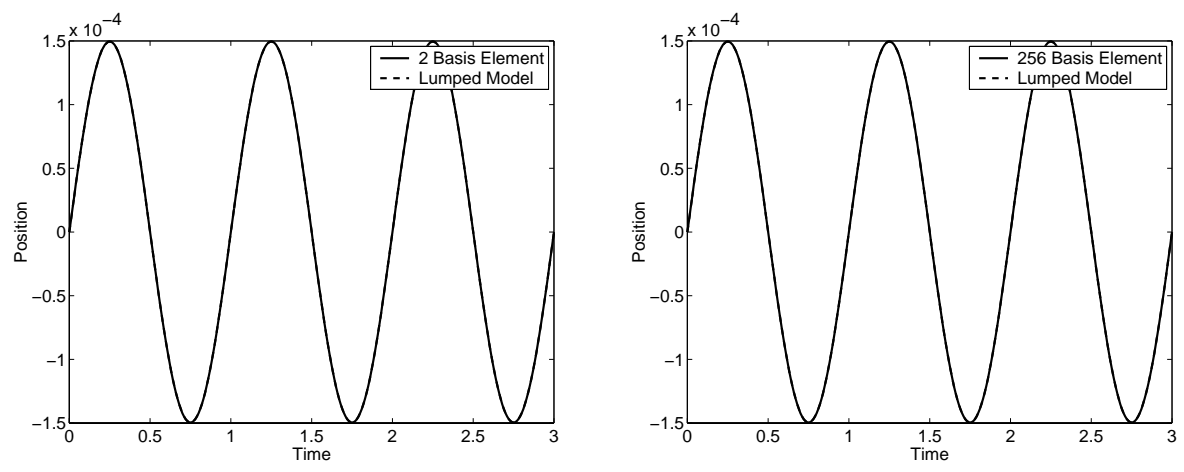

Figure 4: Comparison of the lumped model (13) with the finite element model (10) linearized about the bias level $\left(H_{0}, M_{0}\right)$ with (a) $N=2$, and (b) $N=256$.

Experiments also indicate that for moderate drive levels about a bias $\left(H_{0}, M_{0}\right)$, stresses exhibit an approximately linear dependence on the magnetization but a nonlinear and hysteretic dependence on the field $H$ or current $I$ to the solenoid. This observation motivates us to linearize about the bias level $M_{0}$ to obtain the final model

$$
\begin{gathered}
\ddot{x}+\widehat{c} \dot{x}+\widehat{k} x=\widehat{\zeta}[M(H)](t) \\
x(0)=x_{0} \quad \dot{x}(0)=\dot{x}_{0} .
\end{gathered}
$$

The scalars $\widehat{c}, \widehat{k}$ and $\widehat{\zeta}$ are determined through a fit to the Galerkin approximation (10) or data from the physical device. A least squares fit to the Terfenol-D data reported in [8] yielded the parameter values $\widehat{c}=7.8899 \times 10^{3}$, $\widehat{k}=6.4251 \times 10^{7}$ and $\widehat{\zeta}=1.3724 \times 10^{-2}$. The accuracy of the lumped model (13) for transducer designs having minimal end effects is illustrated in Figure 4 where it is compared with the finite element model (10) linearized about the bias level $\left(H_{0}, M_{0}\right)$. The accuracy of the finite element model for characterizing the physical device is reported in $[7,8]$. Finally, we note that while the model (13) exhibits a linear dependence on $M$, it retains the fully hysteretic and nonlinear dependence on $H$ and $I$ through the relations (6) and (11).

\section{Nonlinear Adaptive Parameter Estimation Algorithms}

The previous section summarized the PDE model developed in [8] and discussed the development of a second order scalar ODE model (13) quantifying the tip displacement generated by an applied field or current in the Terfenol-D transducer depicted in Figure 1a. The material parameters in the magnetization model (6) were initially identified under the assumption of a constant operating temperature. However, in industrial applications the assumption of fixed temperature is often invalid due to Joule heating resulting from eddy currents and ohmic heating in the solenoid. This necessitates the development of nonlinear adaptive identification techniques to update slowly varying material parameters to accommodate the effects of changing temperatures.

In particular, the model parameter $a$ in the nonlinear hysteretic magnetization model (6) has been observed to reflect changes in behavior due to temperature as noted in (4) and the subsequent discussion. Figure 5 illustrates the effect of temperature changes on the $H-M$ relationship quantified by the model (6) for two different values of the parameter $a$. In this initial development, we focus on the development of nonlinear adaptive parameter estimation techniques to update $a$ to accommodate temperature-dependencies.

To accomplish this, we consider the theory in $[17,18]$ and develop modifications required for the magnetostrictive transducer model employed here. To begin, we will summarize the relevant aspect of the nonlinear adaptive parameter estimation algorithms discussed in $[17,18]$. Since the algorithms developed in $[17,18]$ identify nonlinearly occurring parameters in first-order differential equations, we must subsequently extend these concepts in order to develop a nonlinear adaptive parameter estimation algorithm appropriate for second-order ODE systems such as the model (13) developed to quantify transducer behavior in response to nonlinear and hysteretic inputs. 


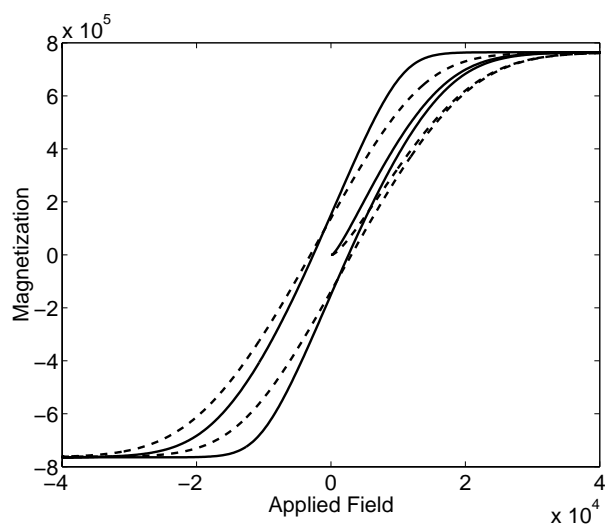

Figure 5: The $H-M$ relation quantified by (6) for two different values of $a$ : (-) $a=7012,(--) a=6300$.

\subsection{Scalar Case}

The nonlinear parameterization techniques developed in $[17,18]$ are based on the assumption that all states are available in systems of the form

$$
\dot{y}=-\lambda y+\nu f(u(t), \theta)
$$

where $\lambda>0$ and $\nu$ are scalars, $\theta \in \Theta$ is an unknown parameter and $\Theta$ is the bounded region in $\mathbb{R}^{m}$. The function $f$ is taken to be a scalar-valued nonlinear function of the input $u(t)$. For example, in the numerical results presented in Section $4, f$ is taken to be the magnetization model $(6), u(t)$ is the applied field or current, and $\theta$ is taken to be the nonlinearly occurring parameter $a$. As motivated by $[17,18]$, we consider the estimation algorithm

$$
\begin{aligned}
\dot{\hat{y}} & =-\lambda\left[\widehat{y}-\epsilon \operatorname{sat}\left(\frac{\tilde{y}}{\epsilon}\right)\right]+f(u, \widehat{\theta})-\nu^{*} \operatorname{sat}\left(\frac{\tilde{y}}{\epsilon}\right) \\
\tilde{y} & =\widehat{y}-y \\
\tilde{y}_{\epsilon} & =\tilde{y}-\epsilon \operatorname{sat}\left(\frac{\tilde{y}}{\epsilon}\right) \\
\dot{\hat{\theta}} & =-\tilde{y}_{\epsilon} \phi^{*}
\end{aligned}
$$

where $\epsilon>0$, sat $(\cdot)$ is a saturation function defined by

$$
\operatorname{sat}(x)=\left\{\begin{aligned}
1, & x \geq 1 \\
x, & |x|<1 \\
-1, & x \leq-1
\end{aligned}\right.
$$

and $\nu^{*}$ and $\phi^{*}$ are the solution of

$$
\begin{aligned}
\nu^{*} & =\min _{\phi \in \mathbb{R}^{m}} \max _{\theta \in \Theta} J(\theta, \phi) \\
\phi^{*} & =\arg \min _{\phi \in \mathbb{R}^{m}} \max _{\theta \in \Theta} J(\theta, \phi) \\
J(\theta, \phi) & =\operatorname{sat}\left(\frac{\tilde{y}}{\epsilon}\right)\left[f(u, \widehat{\theta})-f(u, \theta)-\phi^{T}(\widehat{\theta}-\theta)\right] .
\end{aligned}
$$

We note that when $|\tilde{y}|<\epsilon$, the adaptation of the parameters stops, imposing what is termed a dead-zone. The method will continue to adapt the parameters until the magnitude of the error $\tilde{y}$ is less than the given $\epsilon$.

We consider the min-max algorithm (15) to handle the regions of nonconvexity of $f$ where the gradient method is insufficient as discussed in Section 1. The use of a tuning error $\tilde{y}_{\epsilon}$ rather than a tracking error $\tilde{y}$ ensures continuity of the adaptation as does the use of a saturation function rather than a signum function [18].

Defining $\tilde{\theta}=\widehat{\theta}-\theta$ and $x=\left[\tilde{y}, \tilde{\theta}^{T}\right]^{T}$, it can be shown that the system (14) is stable about $y=0$ by proving that

$$
V=\tilde{y}_{\epsilon}^{2}+\tilde{\theta}^{2}
$$


is a Lyapunov function. To illustrate the manner through which this is established, we summarize details from the original proof in [18].

Summary of Proof from [18]: We first note that

$$
\dot{V}=2 \tilde{y}_{\epsilon} \dot{\tilde{y}}_{\epsilon}+2 \tilde{\tilde{\theta}} \dot{\tilde{\theta}}
$$

If $|\tilde{y}| \leq \epsilon$, then $\tilde{y}_{\epsilon}=0$ so it follow that $\dot{V}=0$. We then need to show that $\dot{V} \leq 0$ if $|\tilde{y}|>\epsilon$. We can represent $\dot{V}$ as

$$
\begin{aligned}
\dot{V} & =2 \tilde{y}_{\epsilon}\left[-\lambda\left(\widehat{y}-\epsilon \operatorname{sat}\left(\frac{\tilde{y}}{\epsilon}\right)\right)+f(u, \widehat{\theta})-\nu^{*} \operatorname{sat}\left(\frac{\tilde{y}}{\epsilon}\right)+\alpha y-f(u, \theta)\right]-2 \tilde{\theta} \tilde{y}_{\epsilon} \phi^{*} \\
& =-2 \lambda \tilde{y}_{\epsilon} \tilde{y}_{\epsilon}+2 \tilde{y}_{\epsilon}\left[f(u, \widehat{\theta})-f(u, \theta)-\tilde{\theta} \phi^{*}-\nu^{*} \operatorname{sat}\left(\frac{\tilde{y}}{\epsilon}\right)\right] \\
& =-2 \lambda \tilde{y}_{\epsilon}^{2}+2 \tilde{y}_{\epsilon}\left[\left(f(u, \widehat{\theta})-f(u, w)-\tilde{\theta} \phi^{*}\right)-\nu^{*} \operatorname{sat}\left(\frac{\tilde{y}}{\epsilon}\right]\right) .
\end{aligned}
$$

If $\tilde{y}>0$, then $\operatorname{sat}\left(\frac{\tilde{y}}{\epsilon}\right)=1$ so we must have

$$
\nu^{*} \geq \operatorname{sat}\left(\frac{\tilde{y}}{\epsilon}\right)\left[f(u, \widehat{\theta})-f(u, \theta)-\tilde{w} \phi^{*}\right] \text { for all } \theta \in \Theta
$$

This implies that we can let

$$
\nu^{*}=\max _{\theta \in \Theta} \operatorname{sat}\left(\frac{\tilde{y}}{\epsilon}\right)\left[f(u, \widehat{\theta})-f(u, \theta)-\tilde{w} \phi^{*}\right] \text { for any } \phi^{*}
$$

The inequality (16) is satisfied by the definition of $\phi^{*}$ and $\nu^{*}$ so that $\dot{V} \leq 0$.

If $\tilde{y}<0$, then $\operatorname{sat}\left(\frac{\tilde{y}}{\epsilon}\right)=-1$ so we must have

$$
\nu^{*} \geq-\left[f(u, \widehat{\theta})-f(u, \theta)-\tilde{\theta} \phi^{*}\right] \text { for all } \theta \in \Theta
$$

or

$$
\nu^{*} \geq \operatorname{sat}\left(\frac{\tilde{y}}{\epsilon}\right)\left[f(u, \widehat{\theta})-f(u, \theta)-\tilde{w} \phi^{*}\right] \text { for all } \theta \in \Theta
$$

Again we can let

$$
\nu^{*}=\max _{\theta \in \Theta} \operatorname{sat}\left(\frac{\tilde{y}}{\epsilon}\right)\left[f(u, \widehat{\theta})-f(u, \theta)-\tilde{w} \phi^{*}\right] \text { for any } \phi^{*} .
$$

By the definition of $\phi^{*}$ and $\nu^{*}$ the inequality (17) is satisfied, hence $\dot{V} \leq 0$.

To implement the method proposed in the system (14), it is necessary to solve the min-max problem (15). To do so, we must construct a concave cover $\bar{F}(\theta)$ and convex cover $\underline{F}(\theta)$ where the covers satisfy

$$
\bar{F}(\theta) \geq f-\widehat{f} \quad \underline{F}(\theta) \leq f-\widehat{f}
$$

with $\widehat{f}=f(u, \widehat{\theta})$. The following definitions and construction are summarized from [18].

Definition 1: A point $\theta^{0} \in \theta_{c}$ if $\theta^{0} \in \Theta$ and

$$
\nabla f_{\theta^{0}}\left(\theta-\theta^{0}\right) \geq f-f^{0}
$$

where $\left.\nabla f_{\theta^{0}} \equiv \frac{\partial f}{\partial \theta}\right|_{\theta^{0}}$ and $f^{0}=f\left(\phi, \theta^{0}\right)$.

Definition 2: $\tilde{\theta}_{c} \equiv \bar{\theta}_{c} \cap \Theta$ where $\bar{\theta}_{c}$ is the complement of $\theta_{c}$. 


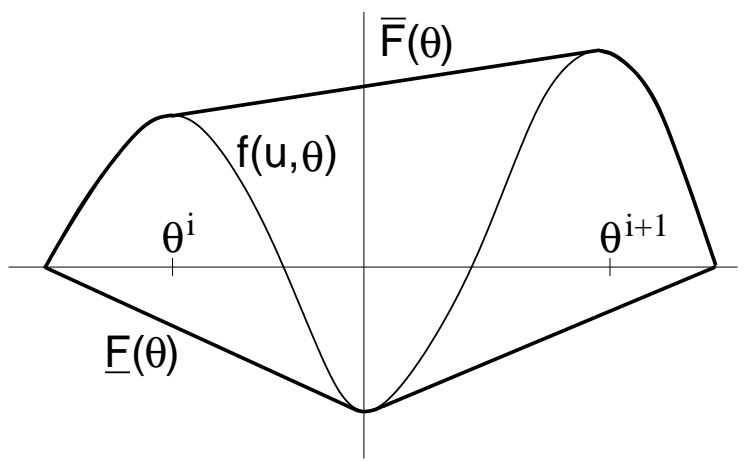

Figure 6: Convex cover $\underline{F}(\theta)$ and concave cover $\bar{F}(\theta)$ of $f(u, \theta)$.

If $f$ is not concave on $\Theta$, then $\tilde{\theta}_{c}$ is given by $\tilde{\theta}_{c}=\left\{\theta^{12}, \theta^{34}, \ldots, \theta^{m n}\right\}$ where $\theta^{i j}=\left[\theta^{i}, \theta^{j}\right], \theta^{j} \geq \theta^{i}$, are the regions where $f$ is not concave. Using Definitions 1 and 2, the concave cover of $f-\widehat{f}$ on $\Theta$ can be subsequently constructed as

$$
\bar{F}(\theta)= \begin{cases}f-\widehat{f}, & \text { for all } \theta \in \theta_{c} \\ \omega^{i j} \theta+c^{i j}, & \text { for all } \theta \in \theta^{i j} \in \tilde{\theta}_{c}\end{cases}
$$

where

$$
\omega^{i j}=\frac{f^{j}-f^{i}}{\theta^{j}-\theta^{i}}, \quad c^{i j}=f^{i}-\hat{f}-\omega^{i j} \theta^{i}, \quad f^{i}=f\left(\phi, \theta^{i}\right) .
$$

Similarly, we construct a convex cover of $f-\widehat{f}$ by defining

$$
\begin{gathered}
\theta_{v} \equiv\left\{\theta^{0} \mid \nabla f_{\theta^{0}}\left(\theta-\theta^{0}\right) \leq f-f^{0}\right\} \\
\tilde{\theta}_{v} \equiv \bar{\theta}_{v} \cap \Theta \\
\underline{F}(\theta)=\left\{\begin{array}{lr}
f-\widehat{f}, & \text { for all } \theta \in \theta_{v} \\
\omega^{i j} \theta+c^{i j}, & \text { for all } \theta \in \theta^{i j} \in \tilde{\theta}_{v} .
\end{array}\right.
\end{gathered}
$$

An illustrative example of a convex and concave cover of a function $f$ is depicted in Figure 6.

Once we have constructed $\underline{F}(\theta)$ and $\bar{F}(\theta)$, a closed form solution to the min-max problem (15) is given by

$$
\begin{aligned}
& \nu^{*}=\bar{F}(\widehat{\theta}) \\
& \phi^{*}=\left\{\begin{array}{c}
\nabla f_{\widehat{\theta}}, \text { if } \widehat{\theta} \in \theta_{c} \\
\omega^{i j}, \text { if } \widehat{\theta} \in \theta^{i j} \in \tilde{\theta}_{c}
\end{array}\right. \\
& \nu^{*}=-\underline{F}(\widehat{\theta}) \\
& \phi^{*}=\left\{\begin{array}{l}
\nabla f_{\widehat{\theta}}, \text { if } \widehat{\theta} \in \theta_{v} \\
\omega^{i j}, \text { if } \widehat{\theta} \in \theta^{i j} \in \tilde{\theta}_{v}
\end{array}\right.
\end{aligned}
$$

A proof that (21) is the solution to (15) can be found in [18].

Having established the stability of the adaptation method by the Lyapunov function, we now seek sufficient conditions which guarantee uniform asymptotic stability of the system (14). We summarize the condition for convergence of the parameters and tracking error in the following theorem presented from [17].

Theorem 1: If for every $t_{1}>t_{0}$, there exists $T_{0}, \epsilon_{0}, \delta_{0}$, and a subinterval $\left[t_{2}, t_{2}+\delta_{0}\right] \in\left[t_{1}, t_{1}+T_{0}\right]$ such that

$$
\beta \int_{t_{2}}^{t_{2}+\delta_{0}} \nu\left[f\left(u, \widehat{\theta}\left(t_{2}\right)\right)-f(u, \theta)\right] d \tau \geq 2 \epsilon+\epsilon_{0}\left\|\tilde{\theta}\left(t_{2}\right)\right\|,
$$

then the origin $y=0$ is uniformly asymptotically stable. 
In Theorem $1, \beta=1$ if $f(u, \widehat{\theta})$ is convex and $\beta=-1$ if $f(u, \widehat{\theta})$ is concave. We note several differences between this condition and the condition for a linear parameterization. First, the sign of the integral is important. The sign is not strictly determined by $f(u, \widehat{\theta})-f(u, \theta)$ but also by the convexity or concavity of $f$ as indicated by $\beta$. This coupling arises from the min-max algorithm and is necessary but not sufficient to ensure that the method will leave the dead zone $|\tilde{y}| \leq \epsilon$. The integral must also be sufficiently large to leave the deadzone, which necessitates the term incorporating $\epsilon$ on the right hand side of (22).

Whereas Theorem 1 places the excitation conditions on $f$, we wish to derive conditions on $u(t)$ since we have some freedom when choosing $u(t)$. Theorem 1 does not give conditions on the input $u$ to satisfy the inequality (22) nor does it guarantee that such an input exists. Inequality (22) includes two components. First, the magnitude of the integrand must be sufficiently large. For a large parameter error, the input must be such that the difference between the function evaluated at the actual parameter and the parameter estimate is adequately large; therefore we desire an input signal which drives the function $f$ to a level where a change in the parameter is most noticeable. Secondly, the integral must be the same sign as $\beta$. This condition states that if $f$ is convex, then the integrand should be positive and, conversely, if $f$ is concave, then the integrand should be negative. The min-max feature of the algorithm provides stability, but an acceptable input must be used to guarantee parameter convergence. Parameter convergence is ensured by updating using the gradient information and we must pick an input signal accordingly.

To ensure parameter convergence, we summarize the persistent excitation conditions developed in [17] for the input $u$.

\section{Persistent Excitation Conditions:}

3.1(a). For a given $\tilde{\theta}, u$ must reverse the sign of the integrand of (22) while keeping the convexity/concavity of $f$ fixed.

or

3.1(b). For a given $\tilde{\theta}, u$ must reverse the convexity/concavity of $f$, while preserving the sign of the integrand in equation (22).

A numerical example illustrating these conditions is provided in Section 4.

\subsection{Vector System}

The nonlinear identification algorithm developed in $[17,18]$ and summarized in Section 3.1 is for scalar differential equations and hence is not directly applicable to higher-order differential equations or systems of differential equations of the type arising in physical applications such as the quantification of hysteresis in a magnetostrictive transducer. In this section, we extend the nonlinear parameter identification algorithm to systems of differential equations. We develop the analysis in the context of the motivating second-order system but note that it is sufficiently general to include higher-order systems.

To extend the method to vector systems, we must redefine several of the variables from the scalar case. Since we want to use the solution to the min-max problem (15), we must ensure that we do not affect that aspect of the formulation.

Consider the identification of nonlinearly occurring parameters in the matrix system

$$
\dot{y}=A y+B f(u, \theta)
$$

where $A \in \mathbb{R}^{2 \times 2}$ and $B \in \mathbb{R}^{2 \times 1}$. We consider the adaptation algorithm

$$
\begin{aligned}
\dot{\hat{y}} & =A \widehat{y}+B\left(f(u, \widehat{\theta})-a^{*} \operatorname{sat}\left(\frac{\tilde{y}_{\epsilon}}{\epsilon}\right)\right) \\
\tilde{y} & =\widehat{y}-y \\
\dot{\hat{\theta}} & =-\gamma \tilde{y}_{\epsilon} \phi^{*} \\
\tilde{y}_{\epsilon} & =B_{2} p_{2} \tilde{y}_{1}+B_{2} p_{3} \tilde{y}_{2}
\end{aligned}
$$


where $p_{2}$ and $p_{3}$ are scalars to be determined, $\gamma>0$ is a scalar gain, $B_{2}$ is the $(2,1)$ component of vector $B$, and $a^{*}$ and $\phi^{*}$ are solutions of the min-max problem

$$
\begin{aligned}
a^{*} & =\min _{\phi \in \mathbb{R}^{m}} \max _{\theta \in \Theta} g(\theta, \phi) \\
\phi^{*} & =\arg \min _{\phi \in \mathbb{R}^{m}} \max _{\theta \in \Theta} g(\theta, \phi) \\
g(\theta, \phi) & =\operatorname{sat}\left(\frac{\tilde{y}_{\epsilon}}{\epsilon}\right)\left[f(u, \widehat{\theta})-f(u, \theta)-\phi^{T}(\widehat{\theta}-\theta)\right] .
\end{aligned}
$$

It is important to note that the solution to the min-max problem (25) is the solution to (15) so this aspect of the adaptive estimation is the same as the scalar case. A notable differences between the matrix system method (25) and the scalar method (15) is the definition of $\tilde{y}_{\epsilon}$. The alteration of $\tilde{y}_{\epsilon}$ is necessary for the Lyapunov candidate utilized for the vector system.

The following theorem ensures the globally asymptotic stability of the adaptive parameter estimation algorithm (24).

Theorem 2: If the vector differential equation (23) is globally asymptotically stable, the adaptive parameter estimation algorithm (24) is globally asymptotically stable.

Proof: We consider the Lyapunov function

$$
V=\gamma \tilde{y}^{T} P \tilde{y}+\tilde{\theta}^{2}
$$

where $P$ is a symmetric positive definite matrix. Taking the derivative of (26) yields

$$
\dot{V}=\gamma \dot{\tilde{y}}^{T} P \tilde{y}+\gamma \tilde{y}^{T} P \dot{\tilde{y}}+2 \tilde{\theta} \dot{\hat{\theta}}
$$

with

$$
\dot{\tilde{y}}=A \tilde{y}+B\left[\hat{f}-f-a^{*} \operatorname{sat}\left(\frac{y_{\epsilon}}{\epsilon}\right)\right] .
$$

Employing the definitions of $\dot{\tilde{y}}$ and $\dot{\hat{\theta}}$, we can write

$$
\dot{V}=\gamma\left(\tilde{y}^{T} A^{T} P \tilde{y}+\tilde{y}^{T} P A \tilde{y}\right)+2 \gamma\left(\left[\hat{f}-f-a^{*} \operatorname{sat}\left(\frac{y_{\epsilon}}{\epsilon}\right)\right] B^{T} P \tilde{y}-\tilde{y} \epsilon \tilde{\theta} \phi^{*}\right) .
$$

Since $A$ is globally asymptotically stable, for any symmetric positive definite matrix $Q$ there exists a symmetric positive definite matrix $P$ such that $A^{T} P+P A=-Q$ (e.g., see [2]). Denoting

$$
P=\left[\begin{array}{ll}
p_{1} & p_{2} \\
p_{2} & p_{3}
\end{array}\right]
$$

we have $B^{T} P \tilde{y}=\omega p_{2} \tilde{y}_{1}+\omega p_{3} \tilde{y}_{2}=\tilde{y}_{\epsilon}$. Substituting this into (27) yields

$$
\dot{V}=-\gamma\left(\tilde{y}^{T} Q \tilde{y}\right)+2 \gamma \tilde{y}_{\epsilon}\left(\hat{f}-f-\tilde{\theta} \phi^{*}-a^{*} \operatorname{sat}\left(\frac{y_{\epsilon}}{\epsilon}\right)\right) .
$$

The proof that $\dot{V}<0$ is completed by utilizing the definitions of $a^{*}$ and $\phi^{*}$ as the solutions of (25) in a manner similar to that employed in the proof in the scalar case. Note that the parameters $p_{2}$ and $p_{3}$ can be determined for any symmetric positive definite choice of $Q$.

Notable differences between the vector parameter estimation algorithm and the scalar algorithm include the lack of a dead-zone and the omission of the $\epsilon \operatorname{sat}\left(\frac{\tilde{y}}{\epsilon}\right)$ term in the definition of $\dot{\hat{y}}$. These alterations are due to the form of the Lyapunov function.

\section{Numerical Examples}

Scalar and vector examples are provided to demonstrate properties of the nonlinear adaptive parameter estimation algorithms. In both examples, the nonlinear function is taken to be the (6) for the magnetization $M$ with the input $u$ being the applied field $H$. As stated previously, the nonlinearly occurring parameter $a(T)$ in the magnetization model reflects changes in the transducer behavior due to temperature changes. We make the assumption that the value 


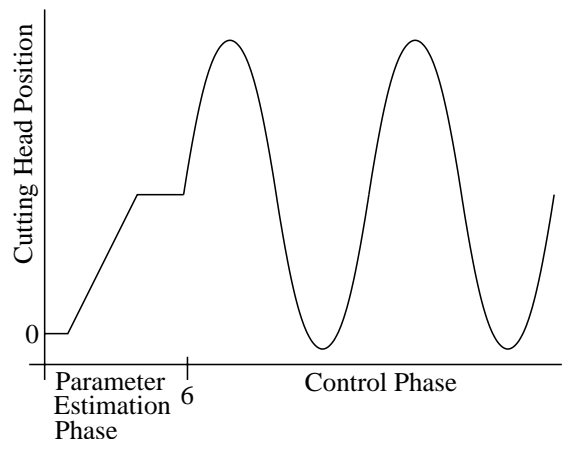

Figure 7: Control trajectory for the milling process and regime during which adaptive estimation occurs.

of $a$ is slowly varying so that the value can assumed to be fixed during the nonlinear parameterization estimation process. During operation, the temperature of the transducer may change and the corresponding value of $a$ can be re-identified during the operation of the transducer.

To provide a specific operating regime, we consider the use of the magnetostrictive transducer depicted in Figure 1a for milling out-of-round automotive products at speeds of $3000 \mathrm{rpm}$ and tolerances of $1 \mu \mathrm{m}$ as described in [26]. The specified trajectory for the cutting head attached to the Terfenol-D rod is depicted in Figure 7. The cutting head starts from rest, is moved to a prescribed position adjacent to the ingot, and then is cycled periodically to produce the desired shape in the automotive product. It is during the initial positioning stage that the nonlinear adaptive identification algorithms are utilized to accommodate thermally-induced changes in the transducer behavior, and it is only during the subsequent periodic cutting stage that the accuracy of $1 \mu \mathrm{m}$ must be maintained.

For both the scalar and vector examples, the parameters in the magnetization model (6) are taken to be $k=$ $400 \mathrm{~A} / \mathrm{m}, \alpha=-0.1, M_{s}=7.65 \times 10^{5} \mathrm{~A} / \mathrm{m}, c=0.18$ and the true value of $a$ is taken as $7012 \mathrm{~A} / \mathrm{m}$. Also, both examples assume that $\widehat{a}$ is bounded with $\widehat{a} \in[6300,7300]$ and $\widehat{a}(0)=6990$. The bounds imposed on $\widehat{a}$ can be determined from experiments under various temperature regimes.

\subsection{Scalar Case}

We illustrate first the scalar case. The dynamics of the system are specified by the differential equation

$$
\dot{y}=-\lambda y+M(H, a)
$$

with the scalar $\lambda$ is taken to be 100 and $M$ specified as the solution to (5) or (6).

One issue in the adaptive parameter estimation algorithm is the construction of inputs $u(t)=H(t)$ which will guarantee persistent excitation. The conditions imposed for excitation prompt the use of a signal that does not cause the function $M$ to change signs. It is observed that a monotonically increasing input provides persistent excitation as well as quick convergence. The input field $H(t)$ was chosen to be a linear function which drives the magnetization to levels where variations in the parameter $a$ are most significant as well as realistically simulating the

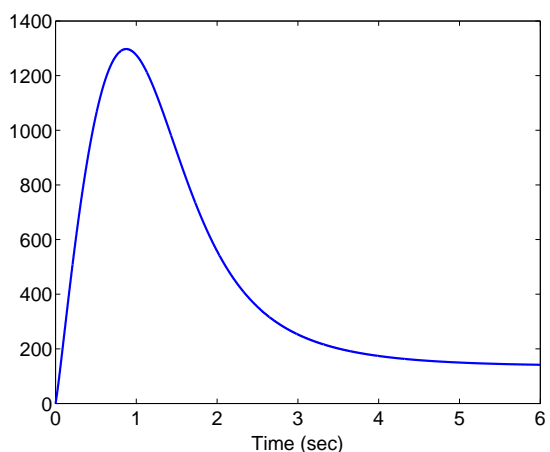

(a)

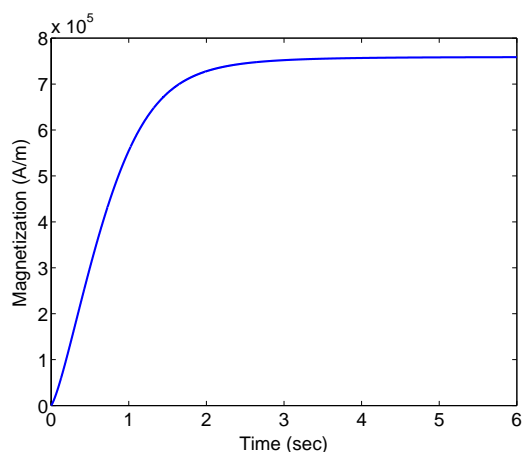

(b)

Figure 8: (a) Value of the integrand in (22), and (b) resulting value of $M(u, \widehat{a})$ provided by (5) or (6). 


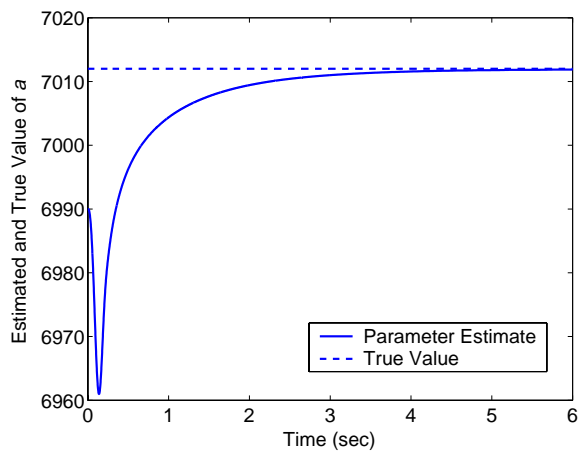

(a)

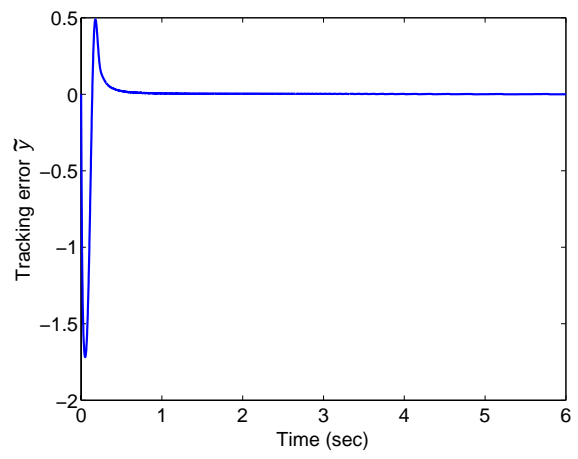

(b)

Figure 9: (a) Parameter estimate $\widehat{a}$ and true value $a=7012 \mathrm{~A} / \mathrm{m}$, and (b) tracking error $\tilde{y}$ for scalar case.

initialization procedure for the milling application. Figure 8a illustrates the integrand of (22) for a given value of $\tilde{\theta}$ to demonstrate that Persistent Excitation Condition 3.1(b) is met. The integrand remains positive while switching the convexity/concavity of the function $M$ as seen in Figure $8 \mathrm{~b}$.

Figure 9 illustrates the capability of the scalar nonlinear parameter estimation method to accurately estimate the unknown parameter $a$. Figure 9a depicts the evolution of the parameter estimate $\widehat{a}$ which quickly converges from the intial value of $a=6990 \mathrm{~A} / \mathrm{m}$ to the true value of $a=7012 \mathrm{~A} / \mathrm{m}$. The tracking error $\tilde{y}$ is plotted in Figure $9 \mathrm{~b}$. The speed of convergence of the estimation algorithm is important since this identification method could potentially be combined with a control technique for online estimation prior to control. We note that the time scales in Figures 8 and 9 are scaled to that in Figure 7 - the convergence rates of both the integrand of (22) and the estimation algorithm are determined by the time needed to cycle the magnetization which can be modified according to the application.

\subsection{Vector System}

We illustrate here the nonlinear adaptive parameter algorithm for a vector system as developed in Section 3.2. The system we consider is the damped spring mass system (13) used to model the transducer dynamics in response to nonlinear and hysteretic inputs with the coefficients specified in Section 2.2. The values of the domain wall model parameters are those specified in Section 4.1. Again, the parameter $a$ is to be determined because of its temperature-dependence. The gain $\gamma$ is taken to be $5 \times 10^{10}$ and $P$ is found utilizing the matrix

$$
Q=\left[\begin{array}{cc}
1 \times 10^{10} & 0 \\
0 & 1 \times 10^{-8}
\end{array}\right]
$$

We employ the same applied magnetic field as in the scalar example.

The convergence of the estimate $\widehat{A}$ for the vector system is illustrated in Figure 10 while Figure 11 illustrates the tracking error of the adaptive system for the vector system in which $\tilde{y}=\left[\tilde{y}_{1}, \tilde{y}_{2}\right]$ denotes the tracking error in the states $y=\left[y_{1}, y_{2}\right]$.

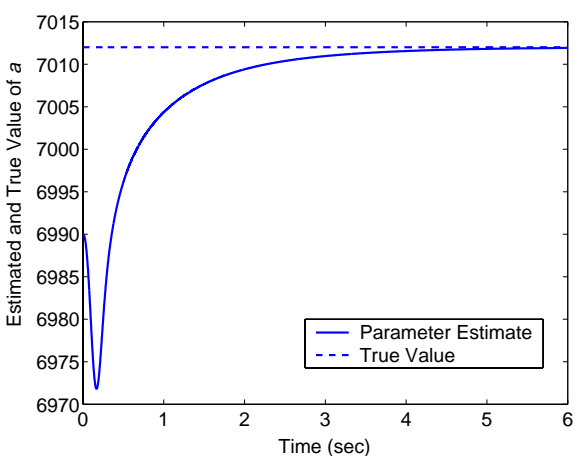

Figure 10: Parameter estimate $\widehat{a}$ and true value $a=7012 \mathrm{~A} / \mathrm{m}$ for the vector system. 


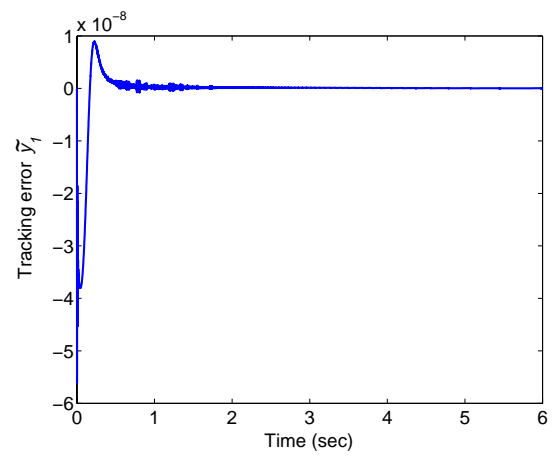

(a)

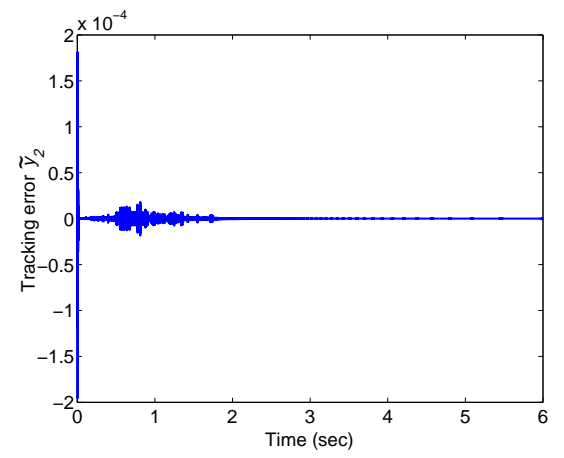

(b)

Figure 11: Tracking errors (a) in the displacement $\tilde{y}_{1}$, and (b) velocity $\tilde{y}_{2}$ for the vector system.

There are a number of numerical issues associated with the implementation of the estimation algorithms for the scalar or vector systems. Inaccuracy in the solution can cause the value of $\tilde{y}$ to have discontinuous jumps from positive to negative values. This phenomena causes the min-max solution to jump between utilizing the convex cover and concave cover which, in turn, can cause highly oscillatory behavior in the parameter updates. The models must be solved numerically and since implicit methods are not feasible because of the unknown forcing function at the next time step, the time step must be sufficiently small to ensure accurate solutions of the model. These issues have been addressed numerically and the experimental implementation of the algorithm is under present investigation.

\section{Concluding Remarks}

The nonlinear parameter identification technique developed in $[17,18]$ has been shown to be effective for identifying and updating a temperature-dependent parameter in the nonlinear domain wall model incorporated as the forcing function in a scalar ODE. We have also extended this parameter identification method to apply to second-order systems. In this latter framework, this adaptive parameter estimation algorithm can be used to estimate and update parameters in a wide range of hysteretic systems including SMAs, ferroelectrics and ferromagnetics. Due to the persistent excitation conditions placed on the input to the hysteresis model, there are potential difficulties in employing the scalar method as a closed-loop nonlinear adaptive control method by considering the nonlinear function in the dynamics to be the composition of an approximate inverse model and the hysteresis system as was done linearly in $[33,34]$. The vector method does not require persistent excitation conditions and hence it may be amenable to incorporation in adaptive control methods utilizing inverse models.

For control designs utilizing this parameter identification method, one can identify the temperature-dependent parameter $a$ in the hysteresis model while the transducer is being initialized. Once the hysteresis model is completely determined, a partial inverse compensation method, e.g., [20] or [26], could be applied. The method in this paper can subsequently be employed to periodically re-identify the parameters in the hysteresis model to ensure accurate modeling as the parameters slowly vary due to the internal heat generated by either the solenoid or surrounding media. This may be more efficient and accurate than current methods of parameter identification, such as least square fits to data, and can be performed online. The next step would be to apply this method to multiple unknown parameters, i.e., $\theta \in \mathbb{R}^{m}$. This method potentially could provide a new method to identify the full set of parameters, $\left\{a, k, c, \alpha, M_{s}\right\}$, simultaneously. While the method can theoretically accommodate multiple parameter identification, there are numerical difficulties in identifying the regions of concavity and convexity. In the one parameter case, we need only to identify linear functions to cover the nonconvex or nonconcave sections of the nonlinear function whereas in a multiple parameter identification bounding hyperplanes are required.

\section{Acknowledgments}

This research was supported in part by the Air Force Office of Scientific Research under the grant AFOSR-F4962001-1-0107. 


\section{References}

[1] A. Adly, I. Mayergoyz and A. Bergqvist, "Preisach modeling of magnetostrictive hysteresis," Journal of Applied Physics, 69(8), pp. 5777-5779, 1991.

[2] B. Anderson and J.B. Moore, Optimal Control: Linear Quadratic Methods, Prentice Hall, Englewood Cliffs, NJ, 1990.

[3] W.F. Brown, Magnetoelastic Interactions. Springer-Verlag, Berlin, 1966.

[4] F.T. Calkins, R.C. Smith and A.B. Flatau, "An energy-based hysteresis model for magnetostrictive transducers," IEEE Transactions on Magnetics, 36(2), pp. 429-439, 2000.

[5] S. Chikazumi, Physics of Ferromagnetism, Clarendon Press, Oxford, 1997.

[6] M.J. Dapino, F.T. Calkins and A.B. Flatau, "Magnetostrictive Devices," Wiley Encyclopedia of Electrical and Electronics Engineering, John G. Webster, Ed., John Wiley and Sons, Inc., Volume 12, pp. 278-305, 1999.

[7] M.J. Dapino, R.C. Smith, L.E. Faidley and A.B. Flatau, "A coupled structural-magnetic strain and stress model for magnetostrictive transducers," Journal of Intelligent Material Systems and Structures, 11(2), pp. 134-152, 2000 .

[8] M.J. Dapino, R.C. Smith and A.B. Flatau, "A structural strain model for magnetostrictive transducers," IEEE Transactions on Magnetics, 36(3), pp. 545-556, 2000.

[9] T. Duering, A. Pelton and D. Stöckel, "An overview of nitinol medical applications," Materials Science and Engineering: A, Vols 273-275, pp. 149-160, 1999.

[10] M.V. Gandhi and B.S. Thompson, Smart Materials and Structures, Chapman and Hall, New York, 1992.

[11] P.K. Hansma, W.B. Elings, O. Marti and C.E. Bracker, "Scanning tunneling microscopy and atomic force microscopy; Applications to biology and technology," Science, 242, pp. 209-242, 1988.

[12] R.D. James and D. Kinderlehrer, "Theory of magnetostriction with applications to $\mathrm{Tb}_{x} \mathrm{Dy}_{1-x} \mathrm{Fe}_{2}$," Philosophical Magazine B, 68(2), pp. 237-274, 1993.

[13] A.P. Jardine, J. Bartley-Cho and J. Flanagan, "Improved design and performance of the SMA torque tube for the DARPA Smart Wing Program," Smart Structures and Materials 1999, Industrial and Commercial Applications of Smart Structures Technologies, Vol. 3674, pp. 260-269, 1999.

[14] D. Jiles, Introduction to Magnetism and Magnetic Materials, Chapman and Hall, New York, 1991.

[15] D.C. Jiles and D.L. Atherton, "Theory of ferromagnetic hysteresis," Journal of Magnetism and Magnetic Materials, 61, pp. 48-60, 1986.

[16] R.A. Kellogg, A.B. Flatau, A.E. Clark, M. Wun-Fogle and T.A. Lograsso, "Temperature and stress dependencies of the magnetic and magnetostrictive properties of $\mathrm{Fe}_{0.81} \mathrm{Ga}_{0.19}$," Journal of Applied Physics, 91(10), pp. 78217823, 2001,

[17] A. Kojic, C. Cao and A. M. Annaswamy, "Parameter Convergence in systems with Convex/Concave Parameterization," Proceedings of the 2000 American Control Conference, pp. 2240-2244, 2000.

[18] A-P Loh, A. M. Annaswamy and F. P. Skantze, "Adaptation in the Presence of a General Nonlinear Parameterization: An Error Model Approach," IEEE Transactions on Automatic Control, 44(9), pp. 1634-1652, 1999.

[19] J.E. Massad and R.C. Smith, "A domain wall model for hysteresis in ferroelastic materials, " Journal of Intelligent Material Systems and Structures, 14(7), pp. 455-571, 2003.

[20] J. Nealis and R.C. Smith, "Partial inverse compensation techniques for linear control design in magnetostrictive transducers," Proceedings of the SPIE, Smart Structures and Materials, 2001, Vol. 4326, pp. 462-473, 2001.

[21] J.B. Restorff, "Magnetostrictive materials and devices," Encyclopedia of Applied Physics, Vol. 9, pp 229-244, 1994. 
[22] J.B. Restorff, H.T. Savage, A.E. Clark and M. Wun-Fogle, "Preisach modeling of hysteresis in Terfenol-D," Journal of Applied Physics, 67(9), pp. 5016-5018, 1996.

[23] J.B. Restorff, M. Wun-Fogle and A.E. Clark, "Temperature and stress dependencies of the magnetostriction in ternary and quaternary Terfenol alloys," Journal of Applied Physics, 87(9), pp. 5786-5788, 2000.

[24] S. Salapaka, A. Sebastian, J.P. Cleveland and M.V. Salapaka, "High Bandwidth nano-positioner: A robust control approach," Review of Scientific Instruments, 73(8), pp.3232-3241, 2002.

[25] S. Seelecke and I. Müller, "Shape memory alloy actuators in smart structures - Modeling and simulation," ASME Applied Mechanics Reviews, to appear.

[26] R.C. Smith, C. Bouton and R. Zrostlik, "Partial and Full Inverse Compensation for Hysteresis in Smart Material Systems," Proceedings of the 2000 American Control Conference, Chicago, IL, June 28-30, pp 2750-2754, 2000.

[27] R.C. Smith, M.J. Dapino and S. Seelecke, "A free energy model for hysteresis in magnetostrictive transducers," Journal of Applied Physics, 93(1), pp. 458-466, 2003.

[28] R.C. Smith and C.L. Hom, "A domain wall theory for ferroelectic hysteresis," Journal of Intelligent Material Systems and Structures, 10(3), pp. 195-213, 1999.

[29] R.C. Smith and J.E. Massad, "A unified methodology for modeling hysteresis in ferroelectric, ferromagnetic and ferroelastic Materials," Proceedings of DETC'01 the ASME Design Engineering Technical Conference and Computers and Information in Engineering Conference, Vol. 6, Pt. B, pp. 1389-1398, 2001.

[30] R.C. Smith and Z. Ounaies, "A domain wall model for hysteresis in piezoelectric materials," Journal of Intelligent Material Systems and Structures, 11(1), pp. 62-79, 2000.

[31] R.C. Smith, S. Seelecke, M.J. Dapino and Z. Ounaies, "A unified model for hysteresis in ferroic materials," Proceedings of the SPIE, Smart Structures and Materials 2003, Volume 5049, pp. 88-99, 2003.

[32] X. Tan, R. Venkataraman and P.S. Krishnaprasad, "Control of hysteresis: Theory and experimental results," Smart Structures and Materials 2001, Modeling, Signal Processing and Control in Smart Structures, SPIE Vol. 4326, pp. 101-112, 2001.

[33] G. Tao and P.V. Kokotović, "Adaptive control of plants with unknown hysteresis," IEEE Transactions on Automatic Control, 40(2), pp. 200-212, 1995.

[34] G. Tao and P.V. Kokotović, Adaptive Control of Systems with Actuator and Sensor Nonlinearities, John Wiley and Sons, New York, 1996. 